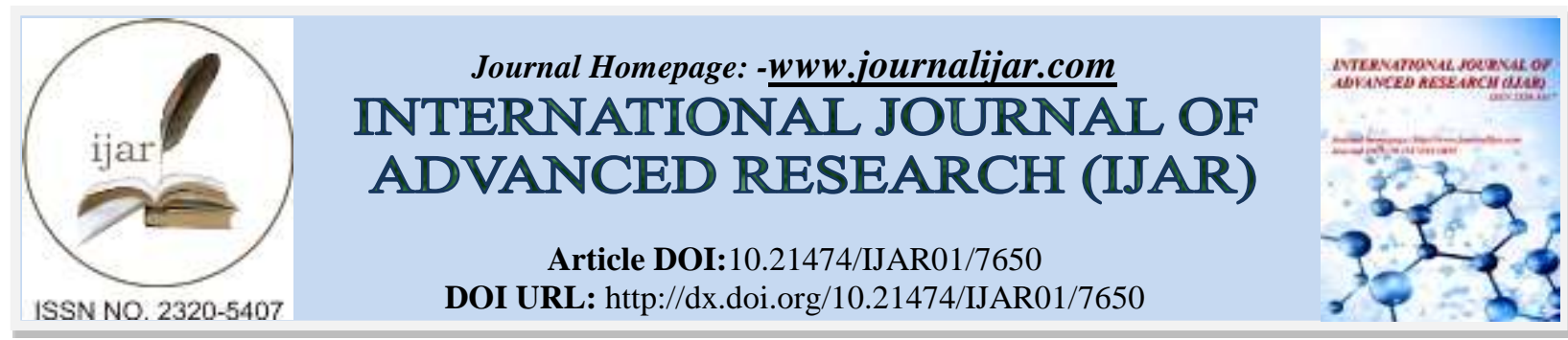

RESEARCH ARTICLE

\title{
VALIDITY OF CONE BEAM COMPUTED TOMOGRAPHY VOXEL DENSITY VALUES: A REVIEW.
}

\section{Kareem S. El-Tabarany, Mona m. Aboulfotouh and Mostafa s. Ashmawy.}

1. B.D.s, faculty of dentistry, ain shams university 2010, cairo, egypt.

2. Professor of oral radiology and diagnostic science faculty of dentistry, ain shams university, cairo, egypt.

3. Lecturer of oral radiology and diagnostic science, faculty of dentistry, ain shams university, cairo, egypt.

\section{Manuscript Info}

Manuscript History

Received: 03 July 2018

Final Accepted: 05 August 2018

Published: September 2018

Keywords: -

Cone-beam computed tomography, grey value, Hounsfield unite, density value, voxel density value.

\section{Abstract}

Introduction: voxel density value in CBCT is used regularly in many clinical aspects such as virtual implant planning. Many researchers studied factors affecting it and their significance. Most of the results obtained are contradicting and /or inconclusive.

Objectives: to compile papers inquiring effect factors affecting voxel grey values and their significance.

Methodology: internet search was done on two databases using MeSH terms. Resulting studies went through two level screening according to predetermined inclusion and exclusion criteria.

Results: A total of 27 studies were included. Total number of factors, which were tested in the included papers, were eighteen.

Conclusion:Significantly affecting factors are (FOV, mA, Objects position inside the FOV, Objects exo-mass, $\mathrm{kVp}$, time between exposure, number of basis, adjacent air to the ROI).Insignificantly affecting factors are (exposure parameters, software, exposure dose, presence of teeth, presence of metallic post in or out the FOV). Inconclusive factors are (Machines model, voxel size, objects mass, receptor type, exposure time). Grey levels in CBCT which is known by Hounsfield Unit of CBCT is not reliable nor repeatable.

Copy Right, IJAR, 2018. All rights reserved.

\section{Introduction: -}

Bone density measurement (BDM) is a valuable tool in several dental applications as preoperative dental implant planning, follow up of periodontal surgeries, surgical reconstruction, ${ }^{1-4}$. As well as in diagnosing many systemic conditions as hyperparathyroidism ${ }^{5-7}$, osteomalacia ${ }^{5,8,9}$, osteoporosis ${ }^{8,10}$, osteopetrosis ${ }^{5,11}$, and blood diseases. ${ }^{5,12,13}$ There are several imaging modalities reported in the literature for BDM with variable degrees of accuracy and reliability; as Dual-energy x-ray absorptiometry (DEXA) ${ }^{14}$, Multi detector computed tomography (MDCT) ${ }^{15}$, Quantitative Computed tomography $(\mathbf{Q C T}){ }^{16}$ and cone beam computed tomography $(\mathbf{C B C T}){ }^{17}$. While MDCT uses the Hounsfield unites (HU) for BDM ${ }^{18}$ with accepted accuracy and reliability ${ }^{19}$, CBCT uses the term Voxel density value (VDV) for BDM, which can be measured at certain point, line, or area on all CBCT reconstructed images ${ }^{20-22}$. Some researchers concluded that CBCT VDVs are accepted as a precise tool for bone density measurements ${ }^{23,24}$. On the other, other authors stated that CBCT VDV are unreliable. ${ }^{25-28}$ 
CBCT is recommended for preoperative implant planning rather than MDCT because of decreased patient absorbed dose ${ }^{29,30}$, integration of different software programmes ${ }^{31,32}$, easy image acquisition ${ }^{32,33}$, availability ${ }^{34}$ and improved patient cooperation ${ }^{22,35}$.

As Olivia Nackaerts ${ }^{36}$ stated that VDVs are not comparable to predetermined standard values because they are subjected to variations due to several factors such as exposure parameters, field of view (FOV), collimation, scattering. ${ }^{20,27,36-38}$ These factors will interfere with the accuracy and reliability of BDM by CBCT, and in turn this might affect the treatment plan or the evaluation process of the treatment outcome. ${ }^{28,36,37}$

Thus, the aim of this present review is to answer a clinically important question which is: "Does CBCT provide accurate and reliable BDM?"

\section{Research methodology}

In order to identify published articles studying the accuracy and/or reliability of CBCT regarding BDM, our search strategy was based on performing two searches, the first used separate keywords, while the second used combined keywords. We searched using the following MeSH terms: "cone beam computed tomography", "bone density" as separate searches then a combined search was performed using "cone beam computed tomography (AND) bone density". We performed our search on Medline and Cochrane libraries. Search was done on April the $21^{\text {st }} 2016$ at 10:21 pm CLT. There were not a date nor language restrictions on any of the searches.

Our search resulted in a total of 92264 studies, 84135 from PubMed and 8129 from Cochrane. Summary of search results are in table 1 .

In order to analyze the search results, we included all articles studying the accuracy and/or reliability of BDM using CBCT. Only researches in which information regarding (1) machine type, (2) exposure parameters and (3) presence of gold standard were included. For studies using quantitative data, agreement between measurements or accuracy of measurements should be present. We excluded (1) studies using CBCT but evaluating the accuracy of another imaging modality (2) non-quantitative studies, (3) studies using CBCT machines not available in the market.

Table 1: -Results for PubMed and Cochrane search engines

\begin{tabular}{|c|c|c|}
\hline $\begin{array}{l}\text { Search } \\
\text { engine }\end{array}$ & Search word & Number of search results \\
\hline \multirow[t]{3}{*}{$\begin{array}{l}\text { Medline } \\
\text { PubMed }\end{array}$} & $\begin{array}{l}\text { Cone-beam computed } \\
\text { tomography }\end{array}$ & 7621 \\
\hline & Bone density & 76226 \\
\hline & $\begin{array}{l}\text { Cone-beam computed } \\
\text { tomography And Bone } \\
\text { density }\end{array}$ & 288 \\
\hline \multirow{16}{*}{$\begin{array}{l}\text { Cochrane } \\
\text { library }\end{array}$} & \multirow{5}{*}{$\begin{array}{l}\text { cone-beam computed } \\
\text { tomography }\end{array}$} & total 220 \\
\hline & & Cochrane Reviews 3 \\
\hline & & Other Reviews 3 \\
\hline & & Trials 208 \\
\hline & & Technology Assessments 6 \\
\hline & \multirow[t]{8}{*}{ Bone density } & total 7895 \\
\hline & & Cochrane Reviews 444 \\
\hline & & Other Reviews 348 \\
\hline & & Trials 6857 \\
\hline & & Methods Studies 10 \\
\hline & & Technology Assessments 91 \\
\hline & & Economic Evaluations 142 \\
\hline & & Cochrane Groups 3 \\
\hline & \multirow{3}{*}{$\begin{array}{l}\text { Cone-beam computed } \\
\text { tomography And Bone } \\
\text { density }\end{array}$} & total 14 \\
\hline & & Cochrane Reviews 1 \\
\hline & & trials 13 \\
\hline
\end{tabular}




\section{Results:-}

All articles were screened by one reviewer on two levels according to the inclusion and exclusion criteria; the initial screening of the articles was conducted using the titles and abstracts, but when these were unclear, the full text was used; this yielded a total of 155 studies. Then the second level was performed by thorough reading and data extraction from the full text; this yielded a total number of 27 article.

The included 27 studies are summarized in Table 2. Studies were gathered according to the searched factor in Table 3

Table 2: -summery of included studies

\begin{tabular}{|c|c|c|c|c|c|c|}
\hline & Title & $1^{\text {st }}$ author & year & journal & $\begin{array}{l}\mathrm{N}^{\mathbf{0}} \text { \& type of } \\
\text { machine(s) }\end{array}$ & $\begin{array}{l}\text { Authors } \\
\text { conclusion }\end{array}$ \\
\hline 1 & $\begin{array}{l}\text { Effects of } \\
\text { image artifacts } \\
\text { on gray-value } \\
\text { density in } \\
\text { limited- } \\
\text { volume cone- } \\
\text { beam } \\
\text { computerized } \\
\text { tomography. }\end{array}$ & Akitoshi Katsumata & 2007 & $\begin{array}{ll}\text { Oral surgery, } & \text { oral } \\
\text { medicine, } & \text { oral } \\
\text { pathology, } & \text { oral } \\
\text { radiology, } & \text { and } \\
\text { endodontics } & \end{array}$ & $\begin{array}{lr}3 \text { machines } & \\
\text { MSCT: } & \text { High } \\
\text { Speed NX } & \\
1^{\text {st }} \text { CBCT: } & 3 \text { DX } \\
\text { Accuitomo } & \text { II } \\
2^{\text {nd }} \text { CBCT: } & 3 \mathrm{DX} \\
\text { FPD } & \end{array}$ & $\begin{array}{l}\text { The intensity of } \\
\text { the discontinuity } \\
\text { artifacts } \\
\text { increased when } \\
\text { more objects } \\
\text { were presented } \\
\text { outside the FOV. }\end{array}$ \\
\hline 2 & $\begin{array}{l}\text { Effect of } \\
\text { object location } \\
\text { on the density } \\
\text { measurement } \\
\text { and } \\
\text { Hounsfield } \\
\text { conversion in } \\
\text { a NewTom 3G } \\
\text { cone beam } \\
\text { computed } \\
\text { tomography } \\
\text { unit }\end{array}$ & MO Lagrave`re & 2008 & $\begin{array}{l}\text { Dentomaxillofacial } \\
\text { Radiology }\end{array}$ & $\begin{array}{lr}1 & \text { machine, } \\
\text { NewTom } & 3 \mathrm{G} \\
\text { Scanner } & \end{array}$ & $\begin{array}{l}\text { the location of an } \\
\text { object within a } \\
\text { CBCT scanner } \\
\text { has a minimal } \\
\text { effect on the } \\
\text { apparent density. }\end{array}$ \\
\hline 3 & $\begin{array}{l}\text { Study of the } \\
\text { scan } \\
\text { uniformity } \\
\text { from an i-CAT } \\
\text { cone beam } \\
\text { computed }\end{array}$ & JA Bryant & 2008 & $\begin{array}{l}\text { Dentomaxillofacial } \\
\text { Radiology }\end{array}$ & 1 machine, i-CAT & $\begin{array}{l}\text { the effects of the } \\
\text { mass and exo- } \\
\text { mass } \\
\text { significant. }\end{array}$ \\
\hline 4 & $\begin{array}{l}\text { The reliability } \\
\text { of computed } \\
\text { tomography } \\
\text { (CT) values } \\
\text { and } \\
\text { dimensional } \\
\text { measurements } \\
\text { of the } \\
\text { oropharyngeal } \\
\text { region using } \\
\text { cone beam } \\
\text { CT: } \\
\text { comparison } \\
\text { with } \\
\text { multidetector } \\
\text { CT } \\
\end{array}$ & A Yamashina & 2008 & $\begin{array}{l}\text { Dentomaxillofacial } \\
\text { Radiology }\end{array}$ & $\begin{array}{lr}2 & \text { machines, } \\
\text { MDCT: } & \text { Aquilin } \\
\text { CBCT: } & \text { CB } \\
\text { Mercuray } & \end{array}$ & $\begin{array}{l}\text { The values of } \\
\text { CBCT differed } \\
\text { markedly from } \\
\text { the HU values } \\
\text { given by a } \\
\text { typical MDCT } \\
\text { system and } \\
\text { became unstable } \\
\text { due to the effects } \\
\text { of adjacent } \\
\text { tissues and had } \\
\text { relatively high } \\
\text { SDs. }\end{array}$ \\
\hline 5 & Relationship & Akitoshi Katsumata & 2009 & Oral surgery, oral & One machine & data \\
\hline
\end{tabular}




\begin{tabular}{|c|c|c|c|c|c|c|}
\hline & $\begin{array}{l}\text { between } \\
\text { density } \\
\text { variability and } \\
\text { imaging } \\
\text { volume } \\
\text { size in cone- } \\
\text { beam } \\
\text { computerized } \\
\text { tomographic } \\
\text { scanning of } \\
\text { the } \\
\text { maxillofacial } \\
\text { region: an in } \\
\text { vitro study }\end{array}$ & & & $\begin{array}{ll}\text { medicine, } & \text { oral } \\
\text { pathology, } & \text { oral } \\
\text { radiology, } & \text { and } \\
\text { endodontics } & \end{array}$ & $\begin{array}{l}\text { CBCT: Alphard } \\
\text { Vega } 3030\end{array}$ & $\begin{array}{l}\text { discontinuity- } \\
\text { related effect was } \\
\text { distinct in } \\
\text { limited-volume } \\
\text { CBCT scanning. }\end{array}$ \\
\hline 6 & $\begin{array}{l}\text { Reliability of } \\
\text { voxel values } \\
\text { from cone- } \\
\text { beam } \\
\text { computed } \\
\text { tomography } \\
\text { for dental use } \\
\text { in evaluating } \\
\text { bone mineral } \\
\text { density } \\
\end{array}$ & Yoshikazu Nomura & 2010 & $\begin{array}{l}\text { clinical oral } \\
\text { implants research }\end{array}$ & $\begin{array}{lr}\text { 2..MDCT } & \text { A } \\
\text { Somatom } & \\
\text { Sensation } & 64 \\
\text { CBCT } & : 3 \mathrm{D} \\
\text { Accuitomo } & \end{array}$ & $\begin{array}{l}\text { This study } \\
\text { confirmed that } \\
\text { there could } \\
\text { be a correlation } \\
\text { between CBCT } \\
\text { voxel values and } \\
\text { BMD, but this } \\
\text { relationship is } \\
\text { not be a linear } \\
\text { one. }\end{array}$ \\
\hline 7 & $\begin{array}{l}\text { Deriving } \\
\text { Hounsfield } \\
\text { units using } \\
\text { grey levels in } \\
\text { cone beam } \\
\text { computed } \\
\text { tomography }\end{array}$ & P Mah & 2010 & $\begin{array}{l}\text { Dentomaxillofacial } \\
\text { Radiology }\end{array}$ & 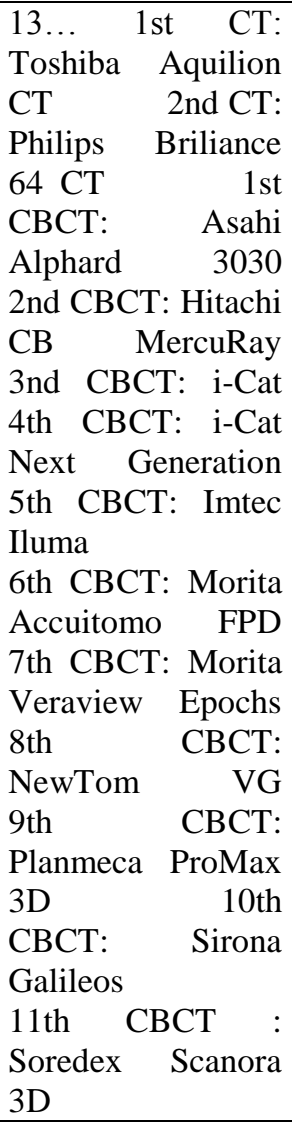 & 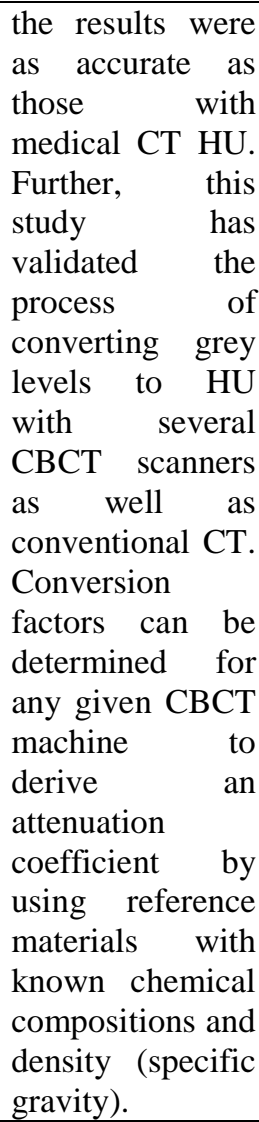 \\
\hline 8 & $\begin{array}{l}\text { Analysis of } \\
\text { intensity } \\
\text { variability in } \\
\text { multislice and }\end{array}$ & Olivia Nackaerts & 2011 & $\begin{array}{l}\text { clinical oral } \\
\text { implants research }\end{array}$ & $\begin{array}{l}\text { 6...MDCT scanner } \\
\text { Somatom } \\
\text { Sensation } \\
\text { 1st CBCT: }\end{array}$ & $\begin{array}{l}\text { The use of } \\
\text { intensity values } \\
\text { in CBCT images } \\
\text { is not reliable, }\end{array}$ \\
\hline
\end{tabular}




\begin{tabular}{|c|c|c|c|c|c|c|}
\hline & $\begin{array}{l}\text { cone beam } \\
\text { computed } \\
\text { tomography }\end{array}$ & & & & $\begin{array}{lr}\text { Accuitomo } & \text { XYZ } \\
\text { 2nd } & \text { CBCT: } \\
\text { Galileos Comforts } \\
\text { 3rd CBCT: Kodak } \\
\text { 9000 } \\
\text { 4th CBCT: Picasso } \\
\text { Duos } \\
\text { 5th } \\
\text { Scanora 3Ds } \\
\end{array}$ & $\begin{array}{l}\text { because the } \\
\text { values are } \\
\text { influenced by } \\
\text { device, imaging } \\
\text { parameters and } \\
\text { positioning. }\end{array}$ \\
\hline 9 & $\begin{array}{l}\text { Qualitative } \\
\text { and } \\
\text { Quantitative } \\
\text { Evaluation of } \\
\text { Bony } \\
\text { Structures } \\
\text { Based on } \\
\text { DICOM } \\
\text { Dataset }\end{array}$ & $\begin{array}{l}\text { Bettina Hohlweg- } \\
\text { Majert }\end{array}$ & 2011 & $\begin{array}{l}\text { journal of oral } \\
\text { maxillofacial } \\
\text { surgery }\end{array}$ & $\begin{array}{l}2 \text { machines, } \\
1^{\text {st }} \text { CBCT: ProMax } \\
\text { 3D } \\
2^{\text {nd }} \text { CBCT: ILUMA } \\
\text { CT }\end{array}$ & $\begin{array}{l}\text { only the CT } \\
\text { finite element } \\
\text { analysis gives } \\
\text { information on } \\
\text { mineral density } \\
\text { and } \\
\text { microarchitecture } \\
\text { of the spongiosa } \\
\text { not CBCT. }\end{array}$ \\
\hline 10 & $\begin{array}{l}\text { Correlation } \\
\text { Between Pixel } \\
\text { Values in a } \\
\text { Cone-Beam } \\
\text { Computed } \\
\text { Tomographic } \\
\text { Scanner and } \\
\text { the Computed } \\
\text { Tomographic } \\
\text { Values in a } \\
\text { Multidetector } \\
\text { Row } \\
\text { Computed } \\
\text { Tomographic } \\
\text { Scanner }\end{array}$ & $\begin{array}{l}\text { Jira } \\
\text { Chindasombatjaroen }\end{array}$ & 2011 & $\begin{array}{l}\text { Journal of } \\
\text { computer assisted } \\
\text { tomography }\end{array}$ & $\begin{array}{l}2 \text { machines, } \\
\text { MDCT LightSpeed } \\
\text { QX/I } \\
\text { CBCT, Alphard } \\
\text { Vega } 3030\end{array}$ & $\begin{array}{l}\text { a high correlation } \\
\text { and linear } \\
\text { relationship } \\
\text { between the CT } \\
\text { values in an } \\
\text { MDCT scanner } \\
\text { and pixel values } \\
\text { in a CBCT } \\
\text { scanner at the } \\
\text { centre of the } \\
\text { FOV were found } \\
\text { for } \\
\text { parameter. }\end{array}$ \\
\hline 11 & $\begin{array}{l}\text { The effect of } \\
\text { surrounding } \\
\text { conditions } \\
\text { on pixel value } \\
\text { of cone beam } \\
\text { computed } \\
\text { tomography }\end{array}$ & Kazuyuki Araki & 2011 & $\begin{array}{l}\text { clinical oral } \\
\text { implants research }\end{array}$ & $\begin{array}{lrr}2 & \text { machines, } \\
\text { MDCT } & \text { GE } & \text { Hi- } \\
\text { Speed } & & \\
\text { CBCT } & & \text { 3D } \\
\text { Accuitomo FPD } 8\end{array}$ & $\begin{array}{l}\text { a high correlation } \\
\text { and linear } \\
\text { relationship } \\
\text { between the CT } \\
\text { values in an } \\
\text { MDCT scanner } \\
\text { and pixel values } \\
\text { in a CBCT } \\
\text { scanner at the } \\
\text { centre of the } \\
\text { FOV were found } \\
\text { for each } \\
\text { parameter. } \\
\text { Therefore, linear } \\
\text { functions can be } \\
\text { used to convert a } \\
\text { pixel value from } \\
\text { the CBCT } \\
\text { machine used in } \\
\text { this study to the } \\
\text { CT values. }\end{array}$ \\
\hline 12 & $\begin{array}{l}\text { Deriving } \\
\text { Hounsfield }\end{array}$ & TE Reeves & 2012 & $\begin{array}{l}\text { Dentomaxillofacial } \\
\text { Radiology }\end{array}$ & $\begin{array}{lr}2 & \text { machines, } \\
1^{\text {st }} & \text { CBCT: Asahi }\end{array}$ & $\begin{array}{l}\text { grey levels taken } \\
\text { from } \quad \text { CBCT }\end{array}$ \\
\hline
\end{tabular}




\begin{tabular}{|c|c|c|c|c|c|c|}
\hline & 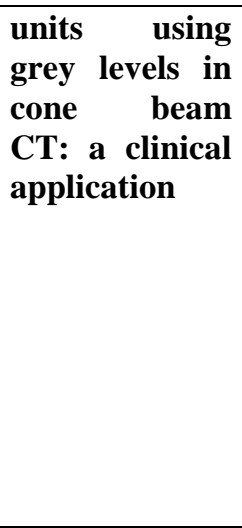 & & & & $\begin{array}{lr}\text { Alphard } & 3030 \\
2^{\text {nd }} & \text { CBCT: } \\
\text { Planmeca } & \text { ProMax } \\
\text { 3D } & \end{array}$ & $\begin{array}{l}\text { scans can be } \\
\text { used to derive } \\
\text { Hounsfield units } \\
\text { in a clinical } \\
\text { environment. } \\
\text { This capability } \\
\text { should solidify } \\
\text { CBCT as the } \\
\text { imaging } \\
\text { modality } \\
\text { choice in dental } \\
\text { implant } \\
\text { placement. }\end{array}$ \\
\hline 13 & $\begin{array}{l}\text { Stability of } \\
\text { voxel values } \\
\text { from cone- } \\
\text { beam } \\
\text { computed } \\
\text { tomography } \\
\text { for dental use } \\
\text { in evaluating } \\
\text { bone mineral } \\
\text { content }\end{array}$ & Yoshikazu Nomura & 2012 & $\begin{array}{l}\text { clinical oral } \\
\text { implants research }\end{array}$ & $\begin{array}{l}2 \\
\text { CBCT, }\end{array}$ & $\begin{array}{l}\text { Pixel value in } \\
\text { CBCT may be } \\
\text { affected } \\
\text { various } \\
\text { conditions such } \\
\text { as beam } \\
\text { hardening and } \\
\text { surrounding } \\
\text { materials, and is } \\
\text { therefore not } \\
\text { reliable as an } \\
\text { index of bone } \\
\text { density. Caution } \\
\text { is essential when } \\
\text { pixel values in } \\
\text { CBCT are used } \\
\text { to estimate bone } \\
\text { density at } \\
\text { potential implant } \\
\text { sites. }\end{array}$ \\
\hline 14 & $\begin{array}{l}\text { Effect of } \\
\text { object location } \\
\text { on the density } \\
\text { measurement } \\
\text { in cone-beam } \\
\text { computed } \\
\text { tomography } \\
\text { versus } \\
\text { multislice } \\
\text { computed } \\
\text { tomography }\end{array}$ & Amir Eskandarloo & 2012 & $\begin{array}{ll}\text { Dental } & \text { research } \\
\text { journal } & \end{array}$ & $\begin{array}{lr}4 & \text { machines, } \\
\text { 1st } & \text { CBCT: } \\
\text { Galileos } & 3 \mathrm{D} \\
2^{\text {nd }} & \text { CBCT: } \\
\text { NewTom } & 3 \mathrm{G} \\
3^{\text {rd }} \text { CBCT: } & \text { Promax } \\
\text { 3D. } & \\
\text { A Multislice CT }\end{array}$ & $\begin{array}{l}\text { the change in } \\
\text { position affects } \\
\text { the density value. } \\
\text { CBCT can show } \\
\text { the clinician the } \\
\text { points with } \\
\text { higher density } \\
\text { and the values } \\
\text { that are exclusive } \\
\text { to each unit, and } \\
\text { are comparable } \\
\text { to Multislice CT, } \\
\text { in cases like the } \\
\text { NewTom } 3 \mathrm{G} \\
\text { type of CBCT. }\end{array}$ \\
\hline 15 & $\begin{array}{l}\text { The accuracy } \\
\text { of CBCT in } \\
\text { measuring } \\
\text { jaws bone } \\
\text { density }\end{array}$ & M. CASSETTA & 2012 & $\begin{array}{l}\text { European Review } \\
\text { for Medical and } \\
\text { Pharmacological } \\
\text { Sciences }\end{array}$ & $\begin{array}{l}1 \text { machine, CBCT } \\
\text { Soredex } \\
\text { SCANORA 3D }\end{array}$ & $\begin{array}{l}\text { the use of a } \\
\text { CBCT to } \\
\text { evaluate the bone } \\
\text { density of jaws is } \\
\text { not useful when } \\
\text { the values are } \\
\text { taken as absolute } \\
\text { values. It does }\end{array}$ \\
\hline
\end{tabular}




\begin{tabular}{|c|c|c|c|c|c|c|}
\hline & & & & & & $\begin{array}{ll}\text { not allow } & \text { an } \\
\text { accurate } & \\
\text { assessment } & \text { of } \\
\text { bone density. } & \\
\end{array}$ \\
\hline 16 & $\begin{array}{l}\text { Influence of } \\
\text { cone beam CT } \\
\text { scanning } \\
\text { parameters on } \\
\text { grey value } \\
\text { measurements } \\
\text { at an implant } \\
\text { site }\end{array}$ & A Parsa & 2013 & $\begin{array}{l}\text { Dentomaxillofacial } \\
\text { Radiology }\end{array}$ & $\begin{array}{lr}3 & \text { machines, } \\
\text { MDCT: } & \text { Philips, } \\
\text { Best } & \\
\text { 1st } & \text { CBCT: } \\
\text { Accuitomo } & 170, \\
\text { Morita } & \\
\text { 2nd } & \text { CBCT: } \\
\text { (NewTom } & 5 \mathrm{G}, \\
\text { Verona } & \end{array}$ & $\begin{array}{l}\text { no statistical } \\
\text { differences in } \\
\text { both CBCT } \\
\text { scanners. } \\
\text { Overall, in the } \\
\text { Accuitomo scans } \\
\text { the FOV, number } \\
\text { of projections } \\
\text { and spatial } \\
\text { resolution had } \\
\text { statistically } \\
\text { significant } \\
\text { influence on } \\
\text { voxel grey value. } \\
\text { FOV and spatial } \\
\text { resolution had a } \\
\text { statistically } \\
\text { significant } \\
\text { influence on } \\
\text { voxel grey value } \\
\text { measurements in } \\
\text { the NewTom } \\
\text { scans, whereas } \\
\text { the effect for } \\
\text { exposure time } \\
\text { and dose was } \\
\text { statistically } \\
\text { insignificant. } \\
\text { Both CBCT } \\
\text { scanners showed } \\
\text { higher grey } \\
\text { values than the } \\
\text { actual } \\
\text { Hounsfield units } \\
\text { derived from } \\
\text { MSCT (mean } 62 \\
\text { HU, standard } \\
\text { deviation } 243 \\
\text { HU). }\end{array}$ \\
\hline 17 & $\begin{array}{l}\text { Prospects and } \\
\text { challenges of } \\
\text { rendering } \\
\text { tissue density } \\
\text { in Hounsfield } \\
\text { units for cone } \\
\text { beam } \\
\text { computed } \\
\text { tomography }\end{array}$ & Roberto Molteni & 2013 & $\begin{array}{ll}\text { Oral surgery, } & \text { oral } \\
\text { medicine, } & \text { oral } \\
\text { pathology, } & \text { oral } \\
\text { radiology, } & \text { and } \\
\text { endodontics } & \end{array}$ & $\begin{array}{lr}2 & \text { machine, } \\
1^{\text {st }} & \text { CBCT } \\
\text { NewTomVGi } \\
2^{\text {nd }} & \text { CBCT } \\
\text { Hyperion } & \text { X9, } \\
\text { MyRay } & \end{array}$ & $\begin{array}{l}\text { CBCT systems } \\
\text { operating at high } \\
\mathrm{kVp} \text { and high } \\
\text { filtration are less } \\
\text { prone to beam } \\
\text { hardening. Beam } \\
\text { hardening } \\
\text { causing an } \\
\text { overall reduction } \\
\text { in the accuracy } \\
\text { of CT numbers } \\
\text { for density } \\
\text { measurements. }\end{array}$ \\
\hline
\end{tabular}




\begin{tabular}{|c|c|c|c|c|c|c|}
\hline & & & & & & $\begin{array}{l}\text { Large FOV } \\
\text { CBCT systems } \\
\text { are less prone to } \\
\text { exomass-related } \\
\text { artifacts. smaller } \\
\text { irradiated and } \\
\text { reconstructed } \\
\text { volumes are less } \\
\text { prone to } \\
\text { inaccurate CT } \\
\text { numbers/HUs, } \\
\text { caused by } \\
\text { scattered } \\
\text { radiation and by } \\
\text { non-ideal } \\
\text { geometry }\end{array}$ \\
\hline 18 & $\begin{array}{l}\text { Computed } \\
\text { gray levels in } \\
\text { multislice and } \\
\text { cone-beam } \\
\text { computed } \\
\text { tomography }\end{array}$ & FabianeAzeredo & 2013 & $\begin{array}{l}\text { American journal } \\
\text { of orthodontics } \\
\text { and dentofacial } \\
\text { orthopaedics }\end{array}$ & $\begin{array}{l}4 \\
\text { MDCT: } r \text { machines, } \\
\text { Dual Slice Helical } \\
\text { CT } \\
1^{\text {st }} \text { CBCT: I-CAT } \\
\text { Imaging Sciences } \\
2^{\text {nd }} \text { CBCT: Sirona } \\
\text { Galileos } \\
3^{\text {rd }} \text { CBCT: Kodak } \\
90003 D\end{array}$ & $\begin{array}{l}\text { 1. Software } \\
\text { programs were } \\
\text { considered } \\
\text { similar for gray } \\
\text { level assessments } \\
\text { in both CT and } \\
\text { CBCT images. } \\
\text { 2. CBCT images } \\
\text { that were not } \\
\text { considered } \\
\text { reliable for } \\
\text { density analysis. }\end{array}$ \\
\hline 19 & \begin{tabular}{ll}
\multicolumn{2}{l}{ Variability of } \\
dental cone \\
beam CT & grey \\
values for \\
density \\
estimations
\end{tabular} & R PAUWELS & 2013 & $\begin{array}{l}\text { The British journal } \\
\text { of radiology }\end{array}$ & 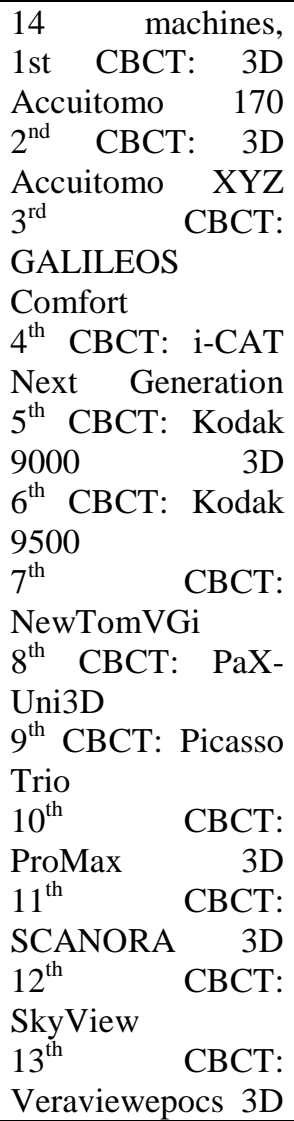 & $\begin{array}{l}\text { most CBCT } \\
\text { devices showed a } \\
\text { good overall } \\
\text { correlation with } \\
\text { CT numbers. The } \\
\text { relatively large } \\
\text { amount of noise } \\
\text { in CBCT may } \\
\text { lead to inaccurate } \\
\text { grey values in } \\
\text { the medium- } \\
\text { density range, } \\
\text { and exo mass can } \\
\text { affect the grey } \\
\text { values inside the } \\
\text { FOV in a non- } \\
\text { uniform way. it } \\
\text { could be possible } \\
\text { to obtain pseudo- } \\
\text { HU from certain } \\
\text { CBCTs. }\end{array}$ \\
\hline
\end{tabular}




\begin{tabular}{|c|c|c|c|c|c|c|}
\hline & & & & & $\begin{array}{l}\text { MSCT Somatom } \\
\text { Sensation } 64\end{array}$ & \\
\hline 20 & $\begin{array}{l}\text { Assessment of } \\
\text { CT numbers } \\
\text { in limited and } \\
\text { medium field- } \\
\text { of-view scans } \\
\text { taken using } \\
\text { Accuitomo } 170 \\
\text { and } \\
\text { Veraviewepocs } \\
\text { 3De cone- } \\
\text { beam } \\
\text { computed } \\
\text { tomography } \\
\text { scanners }\end{array}$ & Matheus L. Oliveira & 2014 & $\begin{array}{l}\text { Imaging Science in } \\
\text { Dentistry }\end{array}$ & $\begin{array}{l}2 \text { machines, } \\
1^{\text {st }} \quad \text { CBCT: } \\
\text { Veraviewepocs } 2^{\text {nd }} \\
\text { CBCT: } 3 \mathrm{De} \\
\text { Accuitomo } 170\end{array}$ & 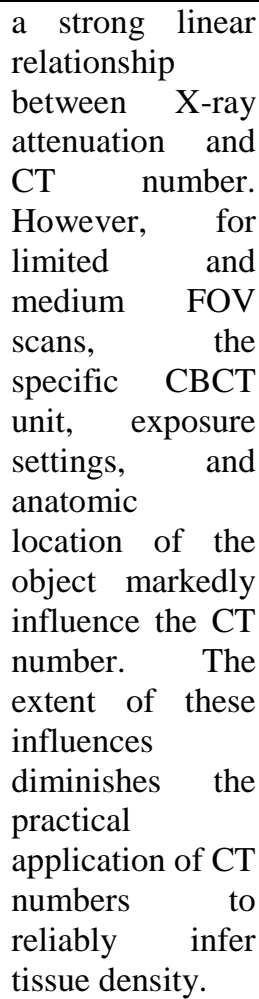 \\
\hline 21 & $\begin{array}{l}\text { Influence of an } \\
\text { object's z-axis } \\
\text { location and } \\
\text { location on the } \\
\text { axial plane on } \\
\text { the voxel value } \\
\text { representation } \\
\text { and } \\
\text { uniformity in } \\
\text { cone beam } \\
\text { computed } \\
\text { tomography }\end{array}$ & Kanako Nishino & 2014 & $\begin{array}{l}\text { Dentomaxillofacial } \\
\text { Radiology }\end{array}$ & $\begin{array}{l}1 \text { machine, } \\
\text { Alphard-3030 }\end{array}$ & $\begin{array}{l}\text { The voxel values } \\
\text { in the Alphard- } \\
3030 \text { changed } \\
\text { based on the z- } \\
\text { axis location of } \\
\text { the radiation } \\
\text { field, even for } \\
\text { the same objects. } \\
\text { In addition, the } \\
\text { voxel values on } \\
\text { the same axial } \\
\text { plane in the } \\
\text { Alphard-3030 } \\
\text { were not uniform } \\
\text { between the } \\
\text { centre and the } \\
\text { off-centre } \\
\text { locations } \\
\text { among the off- } \\
\text { centre locations. }\end{array}$ \\
\hline 22 & $\begin{array}{l}\text { MSCT versus } \\
\text { CBCT: } \\
\text { evaluation of } \\
\text { high- } \\
\text { resolution } \\
\text { acquisition } \\
\text { modes for } \\
\text { dento- } \\
\text { maxillary and }\end{array}$ & $\begin{array}{l}\text { Jean-Philippe } \\
\text { Dillenseger }\end{array}$ & 2014 & $\begin{array}{l}\text { European Society } \\
\text { of Radiology }\end{array}$ & 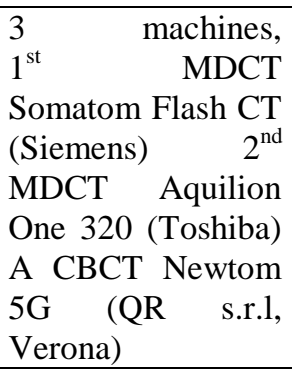 & $\begin{array}{l}\text { CBCT is more } \\
\text { sensitive to } \\
\text { scattered } \\
\text { radiation, which } \\
\text { has an impact on } \\
\text { image } \\
\text { homogeneity. } \\
\text { CBCT currently } \\
\text { it does not }\end{array}$ \\
\hline
\end{tabular}




\begin{tabular}{|c|c|c|c|c|c|c|}
\hline & $\begin{array}{l}\text { skull-base } \\
\text { imaging }\end{array}$ & & & & & surpass MSCT. \\
\hline 23 & $\begin{array}{l}\text { Influence of } \\
\text { exposure } \\
\text { factors on the } \\
\text { variability of } \\
\text { CBCT voxel } \\
\text { values: } \\
\text { phantom } \\
\text { study }\end{array}$ & M L Oliveira & 2014 & $\begin{array}{l}\text { Dentomaxillofacial } \\
\text { Radiology }\end{array}$ & $\begin{array}{l}1 \text { machine, } \\
\text { Picasso-Trio } \\
\text { vatech }\end{array}$ & $\begin{array}{l}\text { exposure factors, } \\
\text { such } \\
\text { milliamperage } \\
\text { and } \mathrm{kVp} \text { did not } \\
\text { influence the } \\
\text { variability in } \\
\text { CBCT voxel } \\
\text { values when the } \\
\text { object exceeded } \\
\text { the FOV size. } \\
\text { However, kVp } \\
\text { reduced this } \\
\text { variability in } \\
\text { scans in which } \\
\text { the FOV } \\
\text { encompassed the } \\
\text { entire object. }\end{array}$ \\
\hline 24 & $\begin{array}{l}\text { Variation in } \\
\text { voxel value } \\
\text { distribution } \\
\text { and effect of } \\
\text { time between } \\
\text { exposures in } \\
\text { six CBCT } \\
\text { units }\end{array}$ & R Spin-Neto & 2014 & $\begin{array}{l}\text { Dentomaxillofacial } \\
\text { Radiology }\end{array}$ & $\begin{array}{l}6 \text { machines, } \\
1^{\text {st }} \text { CBCT: Cranex } \\
\text { 3D } \quad \text { Soredex } \\
2^{\text {nd }} \text { CBCT: Scanora } \\
3^{\text {rd }} \text { CBCT: 3D } \\
\text { NewTom 5G QR } \\
\text { Srl } \\
4^{\text {th }} \text { CBCT: Promax } \\
\text { Dimax 3 Digital } \\
\text { Planmeca } \\
5^{\text {th CBCT: i-CAT }} \\
\text { Imaging Sciences } \\
\text { 3D } \\
6^{\text {th }} \\
\text { Accuitomo FPD80 } \\
\text { Morita }\end{array}$ & $\begin{array}{l}\text { large variation in } \\
\text { grey shade } \\
\text { distribution is } \\
\text { seen in CBCT } \\
\text { data sets. The } \\
\text { pattern of the } \\
\text { variation differs } \\
\text { for some units } \\
\text { depending on the } \\
\text { time interval } \\
\text { between } \\
\text { exposures. }\end{array}$ \\
\hline 25 & $\begin{array}{l}\text { Standardized } \\
\text { method to } \\
\text { quantify the } \\
\text { variation in } \\
\text { voxel value } \\
\text { distribution in } \\
\text { patient- } \\
\text { simulated } \\
\text { CBCT data } \\
\text { sets }\end{array}$ & R Spin-Neto & 2015 & $\begin{array}{l}\text { Dentomaxillofacial } \\
\text { Radiology }\end{array}$ & $\begin{array}{lr}6 & \text { machines, } \\
1^{\text {st }} & \text { CBCT: Cranex } \\
\text { 3D } & \text { Soredex } \\
2^{\text {nd }} \text { CBCT: Scanora } \\
\text { 3D } & \text { Soredex } \\
3^{\text {rd }} & \text { CBCT: } \\
\text { NewTom } & 5 \mathrm{G}, \text { QR } \\
\text { srl } & \\
4^{\text {th }} \text { CBCT: i-CAT } \\
\text { "first generation" } \\
5^{\text {th }} \text { CBCT: } 3 D \\
\text { Accuitomo FPD80 } \\
\text { Morita } 6^{\text {th }} \text { CBCT: } \\
\text { NewTom VG, QR } \\
\text { srl V }\end{array}$ & $\begin{array}{l}\text { discrepancies in } \\
\text { CBCT voxel } \\
\text { value distribution } \\
\text { are seen when } \\
\text { comparing the } \\
\text { initial } \\
\text { examination of } \\
\text { the day with } \\
\text { subsequent } \\
\text { examinations. } \\
\text { Excluding the } \\
\text { initial } \\
\text { examination of } \\
\text { the day, TBE has } \\
\text { no predictable } \\
\text { effect on the } \\
\text { variation of } \\
\text { CBCT-derived } \\
\text { voxel values. }\end{array}$ \\
\hline 26 & $\begin{array}{lr}\text { Use of } & \text { Gray } \\
\text { Values } & \text { in }\end{array}$ & $\begin{array}{l}\text { AndreiaFialho } \\
\text { Rodrigues }\end{array}$ & 2015 & implant dentistry & $\begin{array}{l}2 \text { machines, CBCT } \\
\text { i-CAT }\end{array}$ & $\begin{array}{l}\text { the grey values } \\
\text { obtained in the }\end{array}$ \\
\hline
\end{tabular}




\begin{tabular}{|c|c|c|c|c|c|c|}
\hline & $\begin{array}{l}\text { CBCT and } \\
\text { MSCT } \\
\text { Images for } \\
\text { Determination } \\
\text { of Density: } \\
\text { Influence of } \\
\text { Variation of } \\
\text { FOV Size }\end{array}$ & & & & $\begin{array}{l}\text { MDCT; Somatom } \\
\text { Spirit device }\end{array}$ & $\begin{array}{l}\text { CBCT } \\
\text { examinations are } \\
\text { significantly } \\
\text { influenced by the } \\
\text { FOV size. } \\
\text { Although the } \\
\text { grey values } \\
\text { obtained with } \\
\text { different FOV } \\
\text { sizes in MSCT } \\
\text { have shown } \\
\text { small } \\
\text { differences, these } \\
\text { differences were } \\
\text { statistically } \\
\text { significant. }\end{array}$ \\
\hline 27 & $\begin{array}{l}\text { Reduction of } \\
\text { scatter- } \\
\text { induced image } \\
\text { noise in cone } \\
\text { beam } \\
\text { computed } \\
\text { tomography: } \\
\text { effect of field } \\
\text { of view size } \\
\text { and position }\end{array}$ & Ruben Pauwels & 2016 & $\begin{array}{ll}\text { Oral surgery, } & \text { oral } \\
\text { medicine, } & \text { oral } \\
\text { pathology, } & \text { oral } \\
\text { radiology, } & \text { and } \\
\text { endodontics } & \end{array}$ & $\begin{array}{l}1 \text { machine, 3D } \\
\text { Accuitomo } 170\end{array}$ & $\begin{array}{l}\text { The use of } \\
\text { smaller FOVs } \\
\text { and/or peripheral } \\
\text { FOV position } \\
\text { reduces scatter } \\
\text { and increases } \\
\text { image quality. }\end{array}$ \\
\hline
\end{tabular}


Table 3:-Results of the effect of FOV, $\mathrm{kVp}$ and $\mathrm{mA}$ on the CBCT voxel density values

\begin{tabular}{|l|l|l|l|l|}
\hline & Factor & $\begin{array}{l}\text { Number of tested } \\
\text { papers }\end{array}$ & significant & insignificant \\
\hline 1 & Machine & 13 & 11 & 2 \\
\hline 2 & FOV position & 9 & 9 & 0 \\
\hline 3 & $\begin{array}{l}\text { Objects } \\
\text { inside FOV }\end{array}$ & 5 & 1 \\
\hline 4 & Objects exo-mass & 6 & 6 & 0 \\
\hline 5 & mA & 4 & 2 & 2 \\
\hline 6 & Exposure parameters & 3 & 2 & 1 \\
\hline 7 & Voxel size & 4 & 3 & 1 \\
\hline 8 & Object mass & 4 & 3 & 1 \\
\hline 9 & kVp & 2 & 2 & 0 \\
\hline 10 & Time & 2 & 0 \\
\hline 11 & exposure & Software & 2 & 2 \\
\hline 12 & Number of basis & 2 & 0 & 1 \\
\hline 13 & Receptor type & 1 & 1 & 0 \\
\hline 14 & Adjacent air & 1 & 1 & 0 \\
\hline 15 & Exposure time & 2 & 1 & 1 \\
\hline 16 & Exposure dose & 1 & 1 & 1 \\
\hline 17 & Presence of teeth & 1 & 0 & 1 \\
\hline 18 & $\begin{array}{l}\text { Presence of post } \\
\text { inside or outside FOV }\end{array}$ & 1 & 0 & 1 \\
\hline
\end{tabular}

\section{Discussion:-}

The present review was performed to evaluate validity of voxel density values (VDV) of CBCT. As several authors evaluate pre and post-operative outcome of implant by means of VDV on CBCT. ${ }^{31,39,40}$ while others use VDV in periodontal surgeries and surgical follow up. ${ }^{41-44}$

CBCT provide images comparable to CT but with lower dose. ${ }^{20,} 45$ Several factors affect VDV as kilovoltage, milliamperage, FOV, machines model, exo-mass, and others. ${ }^{36-38,46,47}$ Regarding patient absolute dose the most important factor is FOV. ${ }^{37,48}$

Search was done on PubMed search engine as it has more than 27.3 million records going back to 1966, selectively to the year 1865, and very selectively to 1809 ; about 500,000 new records are added each year. As of the same date, 13.1 million of PubMed's records are listed with their abstracts, and 14.2 million articles have links to full-text. Also it uses MeSH terms and provides a tool to facilitate reaching it "MeSH on demand" 49

Search was done on Cochrane library as it formed to organize medical research findings so as to facilitate evidencebased choices about health interventions faced by health professionals, patients, and policy makers. Cochrane includes 53 review groups that are based at research institutions worldwide. Cochrane has approximately 30,000 volunteer experts from around the world. ${ }^{50}$

Regarding the machines model, total of thirteen papers searched this factor and eleven of them found it significant. $^{24,26-28,36,38,51-57}$

Researches performed by Katsumata et al $2007^{38}$, Nackaerts et al $2011^{36}$, Hohlweg-Majert et al $2011^{52}$, Eskandarloo et al $2012{ }^{55}$, Parsa et al $2013^{53}$, Azeredo et al $2013{ }^{56}$, Pauwels et al $2013^{28}$, Molteni et al $2013{ }^{57}$, Oliveira et al $2014^{54}$, Spin-Neto et al $2014{ }^{27}$ and $2015^{26}$; compared the resultant voxel density values of different CBCT machine models. In all those studies, the exposure parameters were not the same for all the machines used. All of their results showed significant differences in voxel density values between different CBCT machines. However, that difference could not be exclusively because of the machines model, but different exposure parameters as well. 
On the other hand, Mah et al $2010^{51}$ and Reeves et al $2012^{24}$ compared between eleven different CBCT machines. All their results showed change between voxel values more than hundred and twenty-five shades. However, they concluded that machines model was an insignificant factor affecting voxel density values.

All of the studies reviewed regarding machines model used improper scientific methodology. The only research which approached the right methodology was spin-Neto $2015^{26}$ as they had identical parameters in two different machines except for the exposure time that differed by $0.5 \mathrm{sec}$. That factor was also reviewed and revealed inconclusive outcome.

From our point of view, this factor mostly cannot be tested as a single factor as the studies usually did not fix all the parameters between the tested machines as it is mostly impossible to do so.

Machines model can be considered as an inconclusive factor as it was not tested using properly scientific methodology by any study.

Concerning the FOV, total of nine papers discussed the effect of FOV size on voxel grey values. All of them reached the same conclusion that it was a significant factor affecting the voxel density values. ${ }^{28,37,38,53,54,57-60}$

Studies done by Katsumata et al $2007{ }^{38}$ and Oliveira et al $2014{ }^{54}$ tested the FOV as a single factor by fixing all other parameters on the tested machines. Both concluded that it was a significant factor on voxel density values.

Studies of Katsumata et al $2009^{37}$, Parsa et al $2013^{53}$, Molteni et al $2013{ }^{57}$, Pauwels et al $2013^{28}$, Dillenseger et al $2014{ }^{60}$, Rodrigues et al $2015{ }^{59}$ and Pauwels et al $2016^{58}$ searched this factor combined with other factors such as voxel size or machines model; this was because of the unavailability of specific voxel sizes at different FOVs which made the FOV not always a separate factor.Their results were also statistically significant.

Testing the effect of FOV should be done on the same machine so all the exposure parameters can be fixed. From the previous studies, this factor is undoubtedly a significant factor on voxel density values.

As regards the Objects position inside the FOV, total of six researches searched its effect. There was only one research concluded that it has insignificant effect. ${ }^{61}$ All remaining papers found it has significant effect. ${ }^{36,47,54,55,58}$ Studies done by Nackaerts et al $2011^{36}$, Eskandarloo et al $2012^{55}$, Oliveira et al $2014^{54}$, Nishino et al $2014{ }^{47}$, Pauwels et al $2016^{58}$ changed the object position in the FOV of the same machine. All of them, for at least part of their experiments, had all variables fixed except for the tested one. They all concluded that this factor was significant in changing voxel density values.

The results of those studies showed that the effect of object position inside the FOV might be machines and/or exposure parameter dependent as the variations found in densities were not the same.

They did not agree upon the position of least effect on voxel values inside the FOV. One study concluded that it is the peripheral position like the dental $\operatorname{arch}^{58}$. Other study concluded exactly the opposite ${ }^{36}$, as the later concluded that the peripheral position effect is the greatest not the least.

On the other hand Lagrave re et al $2008{ }^{61}$ used single machine and fixed all the variables. Although, difference in voxel values obtained from this study reached hundred and forty-five shades, authors concluded that changes in object locations effect is minimal and insignificant.

In our opinion this factor can easily be tested with proper methodology as all other variables in the machine can be fixed.

Objects exo-mass means the mass of the radiographed object which lies outside the FOV. In vitro this part can affect the voxel value by its presence or absence, by its direction, or by the increase or decrease of the mass. However, clinically its presence or absence, and its increase or decrease are based upon the FOV size. And its direction is based upon the ROI position.

This means that small and medium sized FOV CBCT machines will always have exo-mass.

Total six studies searched this factor ${ }^{37,38,57,62-64}$. All of them concluded it is significant. 
Studies by Katsumata et al $2007^{38}$, Bryant et al $2008^{62}$, Katsumata et al $2009{ }^{37}$, Araki et al $2011^{63}$, Molteni et al $2013^{57}$, Oliveira et al $2014{ }^{64}$ used different combinations of radiographed objects and fixed all exposure parameters in each machine used. All their studies concluded that objects exo-mass affect grey value significantly. And They also specified that the effect is directly proportion to the mass itself.

The study done by Bryant et al $2008^{62}$ concluded that the effect is dependent on its direction and unequal along the FOV. Voxel values was noticed to be high near the exo-mass and decrease gradually to reach the middle which is the most accurate number then continue its decrease till the other side of the FOV with a comb like artifact.

This study also came out with a solution to this issue using algorithms to compensate for the exo-mass effect but only on the machine used there.

The study of Oliviera et al $2014{ }^{64}$ proved that the exo-mass effect on voxel values is directly proportion to exposure parameters. That confirms that the compensation equation found by Bryant et al $2008^{62}$ cannot be generalized to all machines nor every exposure protocol.

Total four studies searched the effect of $\mathbf{m A}$ change on voxel values. Two of them found it is significant, and the other two concluded otherwise. ${ }^{53,64-66}$

From our viewpoint it is considered a tricky factor to test as it can be changed independently in some machines, or in relation to other factors as exposure time or $\mathrm{kVp}$. However, it is in some machines fixed.

Studies done by Chindasombatjaroen et al $2011^{46}$ and Cassetta et al $2012^{66}$ fixed all exposure parameters other than $\mathrm{mA}$ to monitor its change effect. They found its effect is significant on the voxel values.

The study done by Oliveira et al $2014{ }^{64}$ concluded that $\mathrm{mA}$ is insignificantly affected the variation of voxel values. However, their results showed large change in variation of voxel values.

Parsa et al $2013^{53}$ did not separate $\mathrm{mA}$ as a single factor as it was changed along the exposure time. Although exposure time was on the factors affecting voxel values, it was found to be inconclusive.

This study concluded that $\mathrm{mA}$ had insignificant effect on voxel values. However, the changes done here was subtle as it was changed from $0.83 \mathrm{~mA}$ to $0.95 \mathrm{~mA}$, and from $0.59 \mathrm{~mA}$ to 0.60 . those minimal changes should produce minimal if any effect on voxel grey values.

$\mathrm{mA}$ could be seen as debatable conclusion as the final results are tie. But one paper ${ }^{53}$ of the insignificant side tested $\mathrm{mA}$ as a combined factor not a single one. So, it was eliminated. Now, $\mathrm{mA}$ as a factor affecting voxel value can be deduced to be a significant factor.

Regarding the exposure parameters: it is inherently multiple factors and testing it as a single entity might not help to detect which of them actually affecting the voxel values.

$\mathrm{mA}, \mathrm{kVp}, \mathrm{FOV}$, number of basis all are exposure parameters and all were found to be significant. Only one study tested exposure parameters as a single entity ${ }^{67}$.

Nomura et al $2010{ }^{67}$ used four different exposure protocols on one CBCT machine. They interchanged two kVp and two $\mathrm{mA}$ settings. Although they concluded that voxel values of CBCT is not reliable nor repeatable, they also concluded that this factor affected voxel values insignificantly.

Voxel size has limitations in most machines to be changed alone. It is usually attached to FOV, and with lesser extent the rest of the exposure parameters.

Total four researches tested this factor. three of them found it is significant, and one found it otherwise. ${ }^{37,53,57,60}$ Katsumata et al $2009^{37}$, Parsa et al $2013^{53}$ and Dillenseger et al $2015^{60}$ tested voxel size as a combined factor. And their results showed significant changes on voxel values. 
Molteni et al $2013^{57}$ used two voxel sizes; $0.3 \mathrm{~mm}$, and $0.15 \mathrm{~mm}$ to test the effect of this change on voxel values. However, number of basis was not fixed. Here voxel size is a combined factor as well, and it did not show any significant changes on voxel values.

Although three out of four researches results showed it was a significant factor, a final deduction of this factors influence was inconclusive. As, it was not tested as a single factor by any of the studies.

Objects mass is not machine dependent, so it can be easily tested as a single factor. Total four researches tested this factor. three of them found it was significant. ${ }^{51,62-64}$

Bryant et al $2008^{62}$ searched in this factor as a combined factor with objects position. Which was deduced to be a significant factor. They concluded that it was a significant factor.

P mah et al $2010^{51}$ also tested objects mass. The results showed significantly affected voxel values. but FOV of the used CBCT machines were not mentioned. The effect of exo-mass might be present in one or more of the phantoms radiographing set ups. This effect also might have been in some of the machines and not in others.

Araki et al $2011^{63}$ used one CBCT machine and fixed all the parameters except the objects mass. They concluded its effect on voxel values was significant.

Oliveira et al $2014{ }^{64}$ used one CBCT machine in their study. They fixed all exposure parameters and only changed the objects mass by insertion of an implant simulating metallic rod. Their results showed that this factor had an insignificant effect on voxel values.

Two researches which found this factor significant were excluded as they tested it in combination with another factor.

This resulted in a tie. as it only has one paper for each conclusion.

Both studies did not accurately represent clinical situation. Each paper only used one machine, and nothing of the exposure parameters of each machine is remotely related to one another. This factor might be affected by machines model and/or exposure parameter. It needs further researches with proper methodology and on multiple machines to reach a definite conclusion. As its outcome is inconclusive.

kVp is mostly not attached to other parameters. In few machine models it is fixed, others are attached to mA. It can be considered in our opinion an easy factor to test for. Total of two papers searched this factor. One of those studies also investigated the relation between $\mathrm{kVp}$ and exo-mass. Both concluded that it is a significant factor. ${ }^{46,64}$

Chindasombatjaroen et al $2011^{46}$ compared between two kVp 80 and 100 in single CBCT machine and fixed all other parameters. Significant variation occurred to voxel values in response.

Oliveira et al $2014{ }^{64}$ used one CBCT machine and fixed all other parameters. They found that $\mathrm{kVp}$ was a significant factor to voxel values in the presence of exo-mass. Its significance decreases by the absence of exo-mass. Since, most of CBCT machines and most of the protocols used have exo-mass, mostly $\mathrm{kVp}$ is a significant factor in clinical situations to voxel values change.

Regarding Time between exposures there were two papers searched this factor. Both researches were conducted with the same team on two successive years and reached that it was significant. ${ }^{26,27}$

Testing for this factor is easy. As it requires absolute fixation of every variable.

Spin-Neto et al 2014 and $2015^{26,27}$ used six CBCT machines in their studies. They fixed all parameters for each machine. They tested the machines successively with; zero intervals, $15 \mathrm{~min}$ intervals, and 30min intervals. Repetition of those protocols were done on another day

The results were different voxel values between every exposure at the first session and its corresponding one at the second, and between every exposure and its previous one in every group and in every session. 
Each of the tested machine showed different pattern of variation in response to each time intervals.

Time between exposure was found to be a significant factor and its effect was massive, differed from one machine to the other, and was unpredictable even in the same machine.

Concerning the Software used to measure voxel values. This factor is the only one that can be tested for retrospectively on any DICOM file and compare between different programmes on the same file.

Two researches tested that factor. Both concluded its insignificance. ${ }^{56,61}$

Lagrave re et al $2008{ }^{61}$ and Azeredo et al 2013 both tested this using two and five programmes respectively. Results showed insignificant difference on voxel values in both studies.

Number of basis is mostly a combined factor as it is mostly attached to voxel size and/or exposure time and/or rotation angle.

Two papers searched this factor. However, each one concluded exactly the opposite of the other. ${ }^{53,57}$

Parsa et al $2013^{53}$ used multiple exposure sets which made sure that every variable was tested in complete fixation of the others. Number of basis was found to be significant as single factor.

On the other hand Molteni et al $2013{ }^{57}$ tested number of bases effect on voxel value but not as a single factor. Voxel size was not fixed. these results are not conclusive as voxel size itself does not have a conclusion on its significance.

So, number of basis can be considered a significant factor to voxel values.

Only one paper tested receptor type $\mathbf{e}^{38}$. This factor is very hard to be tested as it is always in different machine and mostly different machines possess different sets of exposure parameters.

Katsumata et al $2007^{38}$ used two CBCT machines with different receptors. One was FPD the other was IIT/CCD. Here it is a combined factor as it is in two different machines with two different exposure parameter sets. A significant effect on voxel values was noticed. But it is an inconclusive factor.

Presence of adjacent air to the ROI is an area specific in the radiographed object. Only one research tested this factor. $^{68}$

Yamashina et al $2008{ }^{68}$ tested this factor by using phantom with drilled holes to read the density on the periphery of the holes and away from them.

They found its effect is significant on voxel value.

Exposure time factor is usually a combined factor with voxel size and/or number of basis. Two papers searched this factor one is opposing the other. ${ }^{26,53}$

Parsa et al $2013{ }^{53}$ used two CBCT machines with multiple combinations of exposure parameters sets. Those multiple sets did not totally clarify whether this factor is combined or not.

They concluded that it bestowed an insignificant effect on voxel values.

Spin-Neto ${ }^{26}$ unintentionally tested exposure time between two different machine and results showed significant changes in voxel values. They here tested it surely as combined factor.

It is an inconclusive factor as it was tested as combined factor by both of the testing studies. 
Exposure dose is a factor which is based upon other factors. It is affected by the exposure parameters which in turn have some affect voxel value significantly and others do not. Two studies tested this factor. ${ }^{53,67}$

parsa et al $2013^{53}$ and Nomura et al $2010^{67}$ both tested this factor as a single factor and concluded that its effect is insignificant.

Most of the machines in the market does not provide a direct dose manipulation protocol.

Presence of teeth had one study searched it. It is considered a clinical form of objects mass and/or objects exo-mass according to its position. The only study of it was done by Nomura et al $2013^{69}$ concluded it is an insignificant factor. $^{69}$

Presence of metallic post in or out of FOV is considered the clinical form of objects mass and/or objects exomass. Also, one research tested it and found it is not significant at both stages. The study was done by Nomura et al 2013. ${ }^{69}$

\section{Conclusion: -}

Factors affecting voxel values are 18 factors. they can be divided into three groups; Significantly affecting factors, insignificantly affecting factors, Inconclusive factors.

\section{Significantly affecting factors}

FOV, mA, Objects position inside the FOV, Objects exo-mass, $\mathrm{kVp}$, time between exposure, number of basis, adjacent air to the ROI.

All of the significantly affecting factors bestow an unpredictable effect on voxel values except for the exo-mass. A correcting equation was introduced to reduce the effect of exo-mass on the I Cat machine by JA Bryant ${ }^{62}$.

\section{Insignificantly affecting factors}

exposure parameters, software, exposure dose, presence of teeth, presence of metallic post in or out the FOV. All insignificantly affecting factors were tested by single research paper except for software which was tested by two papers. This necessitates further investigations on those factors.

\section{Inconclusive factors}

Machines model, voxel size, objects mass, receptor type, exposure time.

All of the inconclusive factors need further investigations to reach a proper conclusion.

Finally, voxel values of CBCT are not reliable nor repeatable.

\section{References: -}

1. Valiyaparambil JV, Yamany I, Ortiz D, Shafer DM, Pendrys D, Freilich M, Mallya SM. Bone quality evaluation: comparison of cone beam computed tomography and subjective surgical assessment. Int J Oral Maxillofac Implants 2012; 27: 1271-1277.

2. Mccreadie BR, Goldstein SA. Biomechanics of fracture: is bone mineral density sufficient to assess risk? Journal of bone and mineral research 2000; 15: 2305-2308.

3. Ammann P, Rizzoli R. Bone strength and its determinants. Osteoporos Int 2003; 14 Suppl 3: S13-18.

4. Genant HK, Cooper C, Poor G, Reid I, Ehrlich G, Kanis J, Nordin BC, Barrett-Connor E, Black D, Bonjour J-P. Interim report and recommendations of the World Health Organization task-force for osteoporosis. Osteoporosis International 1999; 10: 259-264.

5. White SC, Pharoah MJ, Baghdady M, Wenzel A, Perschbacher S, Lee L, Jadu F, Ruprecht A, Lam EWN, Carter LC, Benson BW, Murdoch-Kinch CA. partIII interpretation In: Proceedings of the ORAL RADIOLOGY PRINCIPLES AND INTERPRETATION. elsvier mosby, 2014: 271-629.

6. Bilezikian JP, Khan AA, Potts Jr JT, Hyperthyroidism TIWotMoAP. Guidelines for the management of asymptomatic primary hyperparathyroidism: summary statement from the third international workshop. The Journal of Clinical Endocrinology \& Metabolism 2009; 94: 335-339.

7. Silverberg SJ, Locker FG, Bilezikian JP. Vertebral osteopenia: a new indication for surgery in primary hyperparathyroidism. The Journal of Clinical Endocrinology \& Metabolism 1996; 81: 4007-4012.

8. Lerner UH. Bone remodeling in post-menopausal osteoporosis. Journal of Dental Research 2006; 85: 584-595. 
9. El-Desouki MI, Othman SM, Fouda MA. Bone mineral density and bone scintigraphy in adult Saudi female patients with osteomalacia. Saudi medical journal 2004; 25: 355-358.

10. Turner RT, Riggs BL, Spelsberg TC. Skeletal effects of estrogen. Endocrine reviews 1994; 15: $275-300$.

11. Tuukkanen J, Koivukangas A, Jämsä T, Sundquist K, Mackay C, Marks S. Mineral density and bone strength are dissociated in long bones of rat osteopetrotic mutations. Journal of Bone and Mineral Research 2000; 15: 1905-1911.

12. Miller RG, Segal JB, Ashar BH, Leung S, Ahmed S, Siddique S, Rice T, Lanzkron S. High prevalence and correlates of low bone mineral density in young adults with sickle cell disease. American journal of hematology 2006; 81: 236-241.

13. Brennan BM, Mughal Z, Roberts SA, Ward K, Shalet SM, Eden TO, Will AM, Stevens RF, Adams JE. Bone mineral density in childhood survivors of acute lymphoblastic leukemia treated without cranial irradiation. The Journal of Clinical Endocrinology \& Metabolism 2005; 90: 689-694.

14. Blake GM, Fogelman I. An update on dual-energy x-ray absorptiometry. Semin Nucl Med 2010; 40: 62-73.

15. White SC, Pharoah MJ. Oral radiology : principles and interpretation. Mosby St. Louis

16. Bonnick SL. Bone Densitometry in Clinical Practice: Application and Interpretation.

17. White SC. Cone-beam imaging in dentistry. Health Phys 2008; 95: 628-637.

18. Razi T, Niknami M, Alavi Ghazani F. Relationship between Hounsfield Unit in CT Scan and Gray Scale in CBCT. J Dent Res Dent Clin Dent Prospects 2014; 8: 107-110.

19. Lindh C, Nilsson M, Klinge B, Petersson A. Quantitative computed tomography of trabecular bone in the mandible. Dentomaxillofac Radiol 1996; 25: 146-150.

20. Scarfe WC, Farman AG. What is cone-beam CT and how does it work? Dent Clin North Am 2008; 52: 707730, v.

21. Scarfe WC, Angelopoulos C. Maxillofacial Cone Beam Computed Tomography: Principles, Techniques and Clinical Applications. Springer

22. Scarfe WC, Farman AG, Sukovic P. Clinical applications of cone-beam computed tomography in dental practice. Journal-Canadian Dental Association 2006; 72: 75.

23. Suomalainen A, Kiljunen T, Kaser Y, Peltola J, Kortesniemi M. Dosimetry and image quality of four dental cone beam computed tomography scanners compared with multislice computed tomography scanners. Dentomaxillofacial Radiology 2009; 38: 367-378.

24. Reeves TE, Mah P, McDavid WD. Deriving Hounsfield units using grey levels in cone beam CT: a clinical application. Dentomaxillofac Radiol 2012; 41: 500-508.

25. Isoda K, Ayukawa Y, Tsukiyama Y, Sogo M, Matsushita Y, Koyano K. Relationship between the bone density estimated by cone-beam computed tomography and the primary stability of dental implants. Clinical oral implants research 2012; 23: 832-836.

26. Spin-Neto R, Gotfredsen E, Wenzel A. Standardized method to quantify the variation in voxel value distribution in patient-simulated CBCT data sets. Dentomaxillofac Radiol 2015; 44: 20140283.

27. Spin-Neto R, Gotfredsen E, Wenzel A. Variation in voxel value distribution and effect of time between exposures in six CBCT units. Dentomaxillofac Radiol 2014; 43: 20130376.

28. Pauwels R, Nackaerts O, Bellaiche N, Stamatakis H, Tsiklakis K, Walker A, Bosmans H, Bogaerts R, Jacobs R, Horner K. Variability of dental cone beam CT grey values for density estimations. The British journal of radiology 2013; 86: 20120135-20120135.

29. Kau C, Richmond S, Palomo J, Hans M. Current products and practice: three-dimensional cone beam computerized tomography in orthodontics. Journal of orthodontics 2005; 32: 282-293.

30. Carrafiello G, Dizonno M, Colli V, Strocchi S, Taubert SP, Leonardi A, Giorgianni A, Barresi M, Macchi A, Bracchi E. Comparative study of jaws with multislice computed tomography and cone-beam computed tomography. La radiologia medica 2010; 115: 600-611.

31. Liang X, Jacobs R, Hassan B, Li L, Pauwels R, Corpas L, Souza PC, Martens W, Shahbazian M, Alonso A, Lambrichts I. A comparative evaluation of Cone Beam Computed Tomography (CBCT) and Multi-Slice CT (MSCT) Part I. On subjective image quality. Eur J Radiol 2010; 75: 265-269.

32. Suomalainen A. Cone beam computed tomography in oral radiology. Institute of Dentistry, University of Helsinki Helsinki, Finland, Oral Radiology, Helsinki Medical Imaging Center, Helsinki University Central Hospital Helsinki, Finland, 2010.

33. Scarfe WC, Li Z, Aboelmaaty W, Scott S, Farman A. Maxillofacial cone beam computed tomography: essence, elements and steps to interpretation. Australian dental journal 2012; 57: 46-60.

34. Macleod I, Heath N. Cone-beam computed tomography (CBCT) in dental practice. Dent Update 2008; 35: 590592. 
35. O'Neil P. Cone Beam Computed Tomography for General Dentists. Dentistry 2012; 01.

36. Nackaerts O, Maes F, Yan H, Couto Souza P, Pauwels R, Jacobs R. Analysis of intensity variability in multislice and cone beam computed tomography. Clin Oral Implants Res 2011; 22: 873-879.

37. Katsumata A, Hirukawa A, Okumura S, Naitoh M, Fujishita M, Ariji E, Langlais RP. Relationship between density variability and imaging volume size in cone-beam computerized tomographic scanning of the maxillofacial region: an in vitro study. Oral Surgery, Oral Medicine, Oral Pathology, Oral Radiology, and Endodontology 2009; 107: 420-425.

38. Katsumata A, Hirukawa A, Okumura S, Naitoh M, Fujishita M, Ariji E, Langlais RP. Effects of image artifacts on gray-value density in limited-volume cone-beam computerized tomography. Oral Surgery, Oral Medicine, Oral Pathology, Oral Radiology, and Endodontology 2007; 104: 829-836.

39. Atsumi M, Park SH, Wang HL. Methods used to assess implant stability: current status. Int J Oral Maxillofac Implants 2007; 22: 743-754.

40. Hatcher DC, Dial C, Mayorga C. Cone beam CT for pre-surgical assessment of implant sites. CDA 2003; 31 : 825-834.

41. Van der Weijden F, Dell'Acqua F, Slot DE. Alveolar bone dimensional changes of post-extraction sockets in humans: a systematic review. J Clin Periodontol 2009; 36: 1048-1058.

42. Vandenberghe B, Jacobs R, Yang J. Diagnostic validity (or acuity) of 2D CCD versus 3D CBCT-images for assessing periodontal breakdown. Oral Surgery, Oral Medicine, Oral Pathology and Oral Radiology 2007; 104: 395-401.

43. Estrela C, Bueno MR, Leles CR, Azevedo B, Azevedo JR. Accuracy of cone beam computed tomography and panoramic and periapical radiography for detection of apical periodontitis. Journal of endodontics 2008; 34 : 273-279.

44. Zhi-gui M, Lin-feng F. The role of CBCT in evaluation of alveolar bone state during orthodontic-periodontal treatment. Shanghai Journal of Stomatology 2010; 19.

45. Mah JK, Danforth RA, Bumann A, Hatcher D. Radiation absorbed in maxillofacial imaging with a new dental computed tomography device. Oral Surgery, Oral Medicine, Oral Pathology and Oral Radiology 2003; 96: 508513.

46. Chindasombatjaroen J, Kakimoto N, Shimamoto H, Murakami S, Furukawa S. Correlation between pixel values in a cone-beam computed tomographic scanner and the computed tomographic values in a multidetector row computed tomographic scanner. J Comput Assist Tomogr 2011; 35: 662-665.

47. Nishino K, Shimamoto H, Kakimoto N, Tsujimoto T, Chindasombatjaroen J, Murakami S, Furukawa S. Influence of an object's z-axis location and location on the axial plane on the voxel value representation and uniformity in cone beam computed tomography. Oral Surg Oral Med Oral Pathol Oral Radiol 2014; 118: 619624.

48. Hofmann E, Schmid M, Sedlmair M, Banckwitz R, Hirschfelder U, Lell M. Comparative study of image quality and radiation dose of cone beam and low-dose multislice computed tomography--an in-vitro investigation. Clin Oral Investig 2014; 18: 301-311.

49. (2018) pubmed. medline, vol 2018, p scintefic literature search engine

50. (2018) wikipedia. vol 2018

51. Mah P, Reeves TE, McDavid WD. Deriving Hounsfield units using grey levels in cone beam computed tomography. Dentomaxillofac Radiol 2010; 39: 323-335.

52. Hohlweg-Majert B, Pautke C, Deppe H, Metzger MC, Wagner K, Schulze D. Qualitative and quantitative evaluation of bony structures based on DICOM dataset. J Oral Maxillofac Surg 2011; 69: 2763-2770.

53. Parsa A, Ibrahim N, Hassan B, Motroni A, van der Stelt P, Wismeijer D. Influence of cone beam CT scanning parameters on grey value measurements at an implant site. Dentomaxillofac Radiol 2013; 42: 79884780.

54. Oliveira ML, Tosoni GM, Lindsey DH, Mendoza K, Tetradis S, Mallya SM. Assessment of CT numbers in limited and medium field-of-view scans taken using Accuitomo 170 and Veraviewepocs 3De cone-beam computed tomography scanners. Imaging science in dentistry 2014; 44: 279-285.

55. Eskandarloo A, Abdinian M, Salemi F, Hashemzadeh Z, Safaei M. Effect of object location on the density measurement in cone-beam computed tomography versus multislice computed tomography. Dental research journal 2012; 9: S81.

56. Azeredo F, de Menezes LM, Enciso R, Weissheimer A, de Oliveira RB. Computed gray levels in multislice and cone-beam computed tomography. American Journal of Orthodontics and Dentofacial Orthopedics 2013; 144: 147-155.

57. Molteni R. Prospects and challenges of rendering tissue density in Hounsfield units for cone beam computed tomography. Oral Surg Oral Med Oral Pathol Oral Radiol 2013; 116: 105-119. 
58. Pauwels R, Jacobs R, Bogaerts R, Bosmans H, Panmekiate S. Reduction of scatter-induced image noise in cone beam computed tomography: effect of field of view size and position. Oral Surg Oral Med Oral Pathol Oral Radiol 2016; 121: 188-195.

59. Rodrigues AF, da Silva Campos MJ, Chaoubah A, Fraga MR, Vitral RWF. Use of gray values in CBCT and MSCT images for determination of density: influence of variation of FOV size. Implant dentistry 2015; 24: 155-159.

60. Dillenseger JP, Matern JF, Gros CI, Bornert F, Goetz C, Le Minor JM, Constantinesco A, Choquet P. MSCT versus CBCT: evaluation of high-resolution acquisition modes for dento-maxillary and skull-base imaging. Eur Radiol 2015; 25: 505-515.

61. Lagravère M, Carey J, Ben-Zvi M, Packota G, Major P. Effect of object location on the density measurement and Hounsfield conversion in a NewTom 3G cone beam computed tomography unit. Dentomaxillofacial Radiology 2008; 37: 305-308.

62. Bryant JA, Drage NA, Richmond S. Study of the scan uniformity from an i-CAT cone beam computed tomography dental imaging system. Dentomaxillofac Radiol 2008; 37: 365-374.

63. Araki K, Okano T. The effect of surrounding conditions on pixel value of cone beam computed tomography. Clin Oral Implants Res 2013; 24: 862-865.

64. Oliveira ML, Freitas DQ, Ambrosano GM, Haiter-Neto F. Influence of exposure factors on the variability of CBCT voxel values: a phantom study. Dentomaxillofac Radiol 2014; 43: 20140128.

65. Chindasombatjaroen J, Kakimoto N, Murakami S, Maeda Y, Furukawa S. Quantitative analysis of metallic artifacts caused by dental metals: comparison of cone-beam and multi-detector row CT scanners. Oral Radiology 2011; 27: 114-120.

66. Cassetta M, Stefanelli LV, Di Carlo S, Pompa G, Barbato E. The accuracy of CBCT in measuring jaws bone density. Eur Rev Med Pharmacol Sci 2012; 16: 1425-1429.

67. Nomura Y, Watanabe H, Honda E, Kurabayashi T. Reliability of voxel values from cone-beam computed tomography for dental use in evaluating bone mineral density. Clinical Oral Implants Research 2010; 21: 558562.

68. Yamashina A, Tanimoto K, Sutthiprapaporn P, Hayakawa Y. The reliability of computed tomography (CT) values and dimensional measurements of the oropharyngeal region using cone beam CT: comparison with multidetector CT. Dentomaxillofac Radiol 2008; 37: 245-251.

69. Nomura Y, Watanabe H, Shirotsu K, Honda E, Sumi Y, Kurabayshi T. Stability of voxel values from conebeam computed tomography for dental use in evaluating bone mineral content. Clinical oral implants research 2013; 24: 543-548. 